\title{
Guest Editorial for High-Level Parallel Programming and Applications
}

\author{
Clemens Grelck ${ }^{1}$
}

Published online: 11 April 2016

(C) Springer Science+Business Media New York 2016

As processor and system manufacturers increase the amount of both inter- and intra-chip parallelism it becomes crucial to provide the software industry with high-level, abstract, well defined, efficient and effective tools for engineering highquality software for ubiquitous parallel systems. Parallel and distributed programming methodologies are still dominated by low-level techniques such as send/receive message passing or similarly unstructured shared memory mechanisms. High-level parallel programming and its applications offer many potential advantages to this effect and have a key role to play in the scalable exploitation of ubiquitous parallelism.

Since 2001 the series of initially Workshops and more recently Symposia on Highlevel Parallel Programming and Applications (HLPP) has been a forum for researchers developing state-of-the-art concepts, tools and applications for high-level parallel programming. The general emphasis is on software quality, programming productivity and high-level performance models. The 7th International Symposium on High-Level Parallel Programming and Applications was held July 3 and 4, 2014, in the historic Doelenzaal of the University of Amsterdam, right in the historic heart of the city.

HLPP 2014 received 34 submissions, out of which the international programme committee selected 15 contributions for presentation at the symposium and for inclusion in this special issue. The symposium was opened with a keynote by Frank Schlimbach, Intel Corp, entitled Parallelism Through CnC: More Flexibility, Less Pain, that was well received by the 30 participants of the symposium. Our special thanks go to

- Frank Schlimbach for his great keynote,

- the University of Amsterdam for providing a fantastic venue,

Clemens Grelck

c.grelck@uva.nl

1 Informatics Institute, University of Amsterdam, Science Park 904, Amsterdam, The Netherlands 
- Springer Verlag and the International Journal of Parallel Programming for agreeing to publish this special issue and for their continued editorial services and support,

- the HLPP steering committee for accepting our offer to host HLPP 2014 at the University of Amsterdam and for their support from beginning to end,

- the authors of all submitted papers and all participants of HLPP 2014,

- Roy Bakker, Roeland Douma and Jacqueline van der Velde for their organisational support during the symposium,

- the members of the programme committee and all additional reviewers for their crucial work in selecting the best papers for HLPP 2014 and for this special issue.

\section{Programme committee members}

Marco Aldinucci (University of Torino, Italy)

Jost Berthold (University of Copenhagen, Denmark)

Rob Bisseling (Utrecht University, Netherlands)

Murray Cole (University of Edinburgh, United Kingdom)

Joël Falcou (MetaScale / University of Paris-South, France)

Gaétan Hains (University of Paris-East, France)

Zhenjiang Hu (National Institute of Informatics, Japan)

Youry Khmelevsky (University of British Columbia / Okanagan College,

Canada)

Herbert Kuchen (University of Münster, Germany)

Kiminori Matsuzaki (Kochi University of Technology, Japan)

Susanna Pelagatti (University of Pisa, Italy)

Frank Penczek (Intel Corp, Germany)

Tiark Rompf (Oracle Labs / Ecole Polytechnique Fédérale de Lausanne,

Switzerland)

Kostis Sagonas (Uppsala University, Sweden)

Francisco de Sande (University of La Laguna, Spain)

Vijay Saraswat (IBM Thomas J. Watson Research Center, USA)

Sven-Bodo Scholz (Heriot-Watt University, United Kingdom)

\section{Additional reviewers}

Nada Amin (Ecole Polytechnique Fédérale de Lausanne, Switzerland)

Vicente Blanco (University of La Laguna, Spain)

Yuriy Brun (University of Southern California, USA)

Radu Calinescu (University of York, United Kingdom)

Sonia Campa (University of Pisa, Italy)

Andrea Canciani (University of Pisa, Italy)

Emmanuel Chailloux (University Pierre et Marie Curie, France)

Hélène Coullon (University of Orléans, France)

Marco Danelutto (University of Pisa, Italy)

Maurizio Drocco (University of Torino, Italy)

Martin Elsman (University of Copenhagen, Denmark)

Steffen Ernsting (University of Münster, Germany)

Ken Friis Larsen (University of Copenhagen, Denmark)

Alexandra Jimborean (Uppsala University, Sweden) 
Manohar Jonnalagedda (Ecole Polytechnique Fédérale de Lausanne, Switzerland)

Vojin Jovanovic (Ecole Polytechnique Fédérale de Lausanne, Switzerland)

Christos Kartsaklis (Oak Ridge National Laboratory, USA)

David Klaftenegger (Uppsala University, Sweden)

Chong Li (University of Paris-East, France)

Sébastien Limet (University of Orléans, France)

Claudia Misale (University of Torino, Italy)

Akimasa Morihata (Tohoku University, Japan)

Virginia Niculescu (Babes-Bolyai University of Cluj-Napoca, Romania)

Francesco Nidito (University of Pisa, Italy)

Guilherme Peretti Pezzi (University of Torino, Italy)

Raphaël Poss (University of Amsterdam, Netherlands)

Aleksandar Prokopec (Ecole Polytechnique Fédérale de Lausanne, Switzerland)

Shigeyuki Sato (University of Electro-Communications, Japan)

Sandro Stucki (Ecole Polytechnique Fédérale de Lausanne, Switzerland)

Julien Tesson (University of Paris-East, France)

Fabio Tordini (University of Torino, Italy)

Le-Duc Tung (Graduate University for Advanced Studies, Japan)

Kjell Winblad (Uppsala University, Sweden)

Jiangtao Yin (University of Massachusetts Amherst, USA)

Albert-Jan Yzelman (University of Leuven, Belgium)

The Symposia on High-Level Parallel Programming and Applications are well on track. HLPP 2015 will take place at Pisa, Italy, and in 2016 HLPP will be hosted by the University of Münster, Germany.

Amsterdam, Netherlands, January 2015

Clemens Grelck

Organiser of HLPP 2014

Guest editor of this IJPP special issue 